\title{
Abstract \#3: Poster (Other) University of Vermont Medical Laboratory Science: Public Health Laboratory Science Concentration
}

Public Health Laboratories are critical to the US Public Health system, providing surveillance and response to promote better population health outcomes. There has been a long-standing shortage of public health laboratorians and current demographic data indicate this trend will continue. Undergraduate public health laboratory education would prepare competent entry level professionals to enter into the public health workforce. To address this need, in 2006, the University of Vermont's Bachelor of Science in Medical Laboratory Science (MLS) degree program developed a track for students interested in pursuing careers in public health laboratory settings. Students enrolled in the Public Health Laboratory Science (PHLS) concentration complete a modified MLS curriculum with coursework in biostatistics, clinical microbiology, food microbiology, epidemiology and molecular biology. PHLS students apply their learning through a project based, hands-on practicum in a public health laboratory at one of our affiliate sites throughout the Northeast. Projects span a wide array of topics from the development of molecular assays to public health laboratory education related to the identification and prevention of infectious microorganisms. The curriculum meets the requirements for the MLS major with a concentration in PHLS emphasizing microbiology. Students who also complete a clinical microbiology practicum are eligible for the ASCP technologist in microbiology certification exam. Approximately $10 \%$ of our MLS students complete the MLS PHLS concentration. Alumni surveys indicate graduates were very satisfied with the program and all are either employed in the field of public health or continuing their education. 